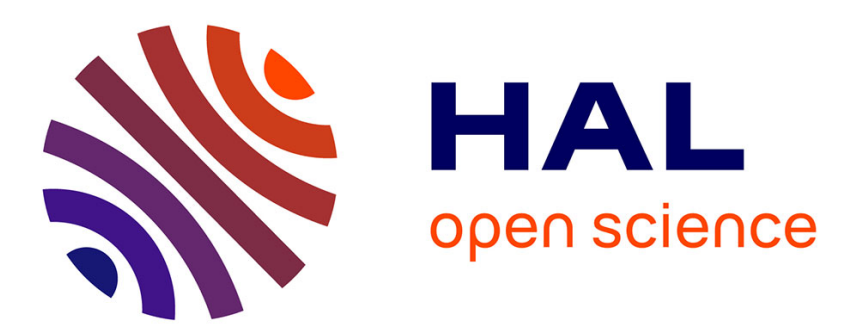

\title{
A new spectral element method with modified Legendre polynomials for modal analysis of lamellar gratings \\ Gérard Gérard Granet
}

\section{To cite this version:}

Gérard Gérard Granet. A new spectral element method with modified Legendre polynomials for modal analysis of lamellar gratings. Journal of the Optical Society of America. A Optics, Image Science, and Vision, 2021, 38 (1), 10.1364/JOSAA.409666 . hal-03040806

\section{HAL Id: hal-03040806 \\ https://hal.science/hal-03040806}

Submitted on 4 Dec 2020

HAL is a multi-disciplinary open access archive for the deposit and dissemination of scientific research documents, whether they are published or not. The documents may come from teaching and research institutions in France or abroad, or from public or private research centers.
L'archive ouverte pluridisciplinaire HAL, est destinée au dépôt et à la diffusion de documents scientifiques de niveau recherche, publiés ou non, émanant des établissements d'enseignement et de recherche français ou étrangers, des laboratoires publics ou privés. 


\title{
A new spectral element method with modified Legendre polynomials for modal analysis of lamellar gratings.
}

\author{
GÉRARD GRANET* \\ Université Clermont Auvergne, CNRS, SIGMA Clermont, Institut Pascal, F-63000 Clermont-Ferrand, France \\ gerard.granet@uca.fr
}

December 4, 2020

\begin{abstract}
We report on the derivation of a spectral element method the originality of which comes from the use of a hierarchical basis built with modified Legendre polynomials. We restrict our work to the TM polarization which is the most challenging. The validation and the convergence is carefully checked for metallic dielectric gratings. The method is shown to be highly efficient and remains stable for huge truncation numbers. All the necessary information is given so that non-specialists can implement the method.
\end{abstract}

\section{INTRODUCTION}

During the last decade, plasmonics has taken an increasingly important part in optics. In this context, dielectric metallic structures, whether periodic or not, play a fundamental role because they support plasmons which correspond to electromagnetic fields with a very fast spatial variation and local huge enhancement. These latter basic properties make the design of plasmonic structures very challenging for optical engineers regardless of the numerical method used. For translation invariant structures, modal methods and mode matching techniques are very interesting in that they avoid discretization along the axis of invariance. $\mathrm{Nu}$ merical modal methods differ in the choice of expansion and test functions. One can distinguish full domain functions or sub-domain functions according to whether they are defined on the whole domain or only on some part of it. For periodic problems, the most popular method is certainly the well-known Fourier Modal Method (FMM) [1] in which expansion and test functions are pseudo periodic functions. On the contrary, using sub-domain expansions within each domain that constitute the structure allows to express rigorously the different continuity relations that determine the eigenvalue problem and thus leads to exponential convergence for the eigenvalues and eigenvectors. The polynomial modal method [2] is one such method. It was revisited in [3], [4] and [5]. It was shown to outperform all other numerical modal methods for dielectric metallic structures. However, its implementation may scare those who are not familiar

\footnotetext{
${ }^{*}$ Corresponding author
}

with numerical methods. Due to locality of basis functions, the numerical polynomial modal methods look quite similar to finite-element based methods. The aim of the present paper is precisely to reformulate it in the frame work of the Spectral Element Method (SEM) and to make it easy for every one. The detailed numerical theory and implementation are given in section II. Numerical results are shown in section III. We restrict our analysis to TM polarized waves and apply it to metallic gratings in the optical domain. This limited area of investigation is actually not a limitation. Indeed all the experience accumulated on the grating theory shows that if a method works well in this particular case, it also works in all other cases [6]. The converse is not true.

\section{PResentation of The PROBlem}

The lamellar diffraction problem is depicted in Fig 1 The grating is illuminated from a homogeneous medium with optical index $n_{1}$ by a monochromatic plane wave with a wavelength $\lambda$ and an angular frequency $\omega$. The wave vector $\mathbf{k}$ forms an angle $\theta$ with respect to the z-axis. Hence its Cartesian coordinates are $k_{x}=k \alpha_{0}=k n_{1} \sin (\theta)$ and $k_{z}=-k n_{1} \cos (\theta)$ with $k=2 \pi / \lambda$ the wavenumber. The $\exp (i \omega t)$ time dependence will be omitted throughout this paper. The grating layer with thickness $h$ is characterized by a piecewise homogeneous permittivity function $\varepsilon(x)$ that is periodic in the $\mathrm{x}$ direction with period $d$. The general solution is a linear combination of two fundamental polarizations: the TE polarization and the TM polarization whose non-null components of the electromagnetic field are 


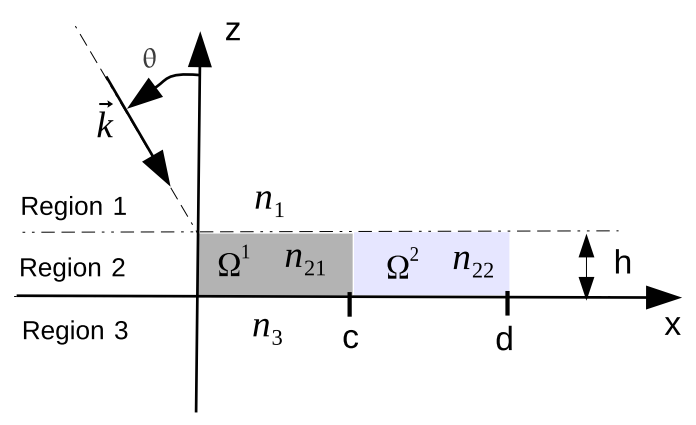

Figure 1: Typical lamellar grating.

$H_{z}, H_{x}, E_{y}$ and $E_{z}, E_{x}, H_{y}$ respectively. We restrict our analysis to the TM polarization which is known to be the most challenging. The difficulty comes from the fact that for some combinations of wavelengths and material, some field components happen to be very confined to the interfaces and others undergo huge discontinuities. In each region, which we refer to by the subscript $l, l=1,2,3, H_{y}$ and $E_{x}$ can be represented by the superposition of forward and backward eigenmodes:

$$
\begin{aligned}
& H_{y l}(x, z)=\sum_{n=1}^{N} A_{l n}^{ \pm} \exp \left(\mp i \gamma_{l n} z\right) \psi_{l n}(x) \\
& E_{x l}(x, z)=\frac{1}{\omega \varepsilon_{l}(x)} \sum_{n=1}^{N} \pm \gamma_{l} A_{l n}^{ \pm} \exp \left(\mp i \gamma_{l n} z\right) \psi_{l n}(x)
\end{aligned}
$$

where $A_{l n}^{+}$and $A_{l n}^{-}$are constant amplitudes and $\gamma_{l n}$ and $\psi_{l n}$ are modal eigenvalues and modal eigenfuntions that are determined by the boundary-value problem:

$$
\begin{gathered}
\left(\frac{d}{d x} \frac{1}{\varepsilon(x)} \frac{d}{d x}+k^{2}\right) \psi_{\ln }(x)=\gamma_{\ln }^{2} \frac{1}{\varepsilon(x)} \psi_{\ln }(x) \\
\psi_{\ln }\left(c^{-}\right)=\psi_{\ln }\left(c^{+}\right) \\
\psi_{\ln }(x+d)=\tau \psi_{\ln }(x) \\
p_{l}^{-} \frac{d \psi_{\ln }}{d x}\left(c^{-}\right)=p_{l}^{+} \frac{d \psi_{\ln }}{d x}\left(c^{+}\right) \\
p_{l}^{+} \frac{d \psi_{\ln }}{d x}(d)=\tau p_{l}^{-} \frac{d \psi_{\ln }}{d x}(0)
\end{gathered}
$$

with $\tau=\exp \left(-i k \alpha_{0} d\right)$ and $p_{l}^{-}=p_{l}^{+}=1$ except in the grating region where we have $p_{2}^{-}=1 / n_{21}^{2}$ and $p_{2}^{+}=1 / n_{22}^{2}$. The eigenvalues are deduced from their square number by:

$$
\gamma_{l n}=\left\{\begin{array}{l}
\sqrt{\gamma_{l q}^{2}} \text { if } \gamma_{l q}^{2} \in R^{+} \\
-i \sqrt{-\gamma_{l q}^{2}} \text { if } \gamma_{l q}^{2} \in R^{-} \\
\sqrt{\gamma_{l q}^{2}} \text { with negative imaginary part if } \gamma_{l q}^{2} \in C
\end{array}\right.
$$

We have to compute the reflection and transmission amplitude coefficients $A_{1 n}^{+}$and $A_{3 n}^{-}$which in turn determine the diffraction efficiencies. Assuming that the unit amplitude incident plane wave corresponds to eigenvector $\psi_{10}$, the reflection and transmission efficiencies $R_{q}, T_{q}$ are given by:

$$
\begin{aligned}
& R_{q}=\frac{\gamma_{1 q}}{\gamma_{10}}\left|A_{1 q}^{+}\right|^{2} \\
& T_{q}=\frac{n_{1}^{2}}{n_{3}^{2}} \frac{\gamma_{3 q}}{\gamma_{10}}\left|A_{3 q}^{-}\right|^{2}
\end{aligned}
$$

The determination of the constant coefficients requires the knowledge of $H_{y}$ and $E_{x}$ in every region. $H_{y l}$ is obtained as a linear combination of the eigenvectors of (2) whereas $E_{x l}$ is deduced from the $H_{y l}$ thanks to (1b). The next section is devoted to the numerical derivation of $H_{y}$ and $E_{x}$.

\section{NUMERICAL SOLUTION}

\section{i. Eigenvalue eigenvector system}

\section{i.1 Weak form of the Helmholtz equation}

The numerical solution follows the well-known steps of the finite elements method [8] [9]. Firstly, the differential equation (2) is turned into an integral equation by multiplying it by some continuous function $\phi(x)$ and integrating it on computational domain $\Omega$ :

$$
\begin{array}{r}
\int_{\Omega} \phi(x) \frac{d}{d x} \frac{1}{\varepsilon(x)} \frac{d}{d x} \psi(x) d x+k^{2} \int_{\Omega} \phi(x) \psi(x) d x= \\
\gamma^{2} \int_{\Omega} \phi(x) \frac{1}{\varepsilon(x)} \psi(x) d x
\end{array}
$$

Secondly, in order to deal with the discontinuity of $\varepsilon(x)$, the whole domain is partitioned into a finite number $Q$ of subdomains $\Omega^{j}$. Let the position of the vertical interfaces be denoted by $x_{j}, j=0, \cdots, Q$, that is $\Omega^{j}=\left\{x \mid x \in\left[x_{j-1}, x_{j}\right]\right\}$. The integral over domain $\Omega$ is obtained by summation of integrals over elements $\Omega^{j}$. Let us consider the first term of the left hand-side of (6) calculated on element $\Omega^{j}$ and let us integrate it by parts. We get:

$$
\begin{aligned}
\int_{\Omega^{j}} \phi(x) \frac{d}{d x} \frac{1}{\varepsilon(x)} \frac{d}{d x} \psi(x) d x & =I_{j+1}-I_{j} \\
& -\int_{\Omega^{j}} \frac{d}{d x} \phi(x) \frac{1}{\varepsilon(x)} \frac{d}{d x} \psi(x) d x
\end{aligned}
$$

with

$$
\begin{aligned}
& I_{j+1}=\left.\phi\left(x_{j+1}\right) \frac{1}{\varepsilon\left(x_{j+1}\right)} \frac{d}{d x} \psi(x)\right|_{x=x_{j+1}} \\
& I_{j}=\left.\phi\left(x_{j}\right) \frac{1}{\varepsilon\left(x_{j}\right)} \frac{d}{d x} \psi(x)\right|_{x=x_{j}}
\end{aligned}
$$


We recognize $E_{z}$ in $\frac{1}{\varepsilon(x)} \frac{d}{d x} \psi(x)$ which is continuous at $x=x_{j}, \quad j=1, \cdots, Q-1$. Hence (6) writes:

$$
\sum_{q=1}^{Q} U^{q}+V^{q}+I_{N}-I_{0}=\sum_{q=1}^{Q} W^{q}
$$

with

$$
\begin{gathered}
U^{q}=\int_{x_{q-1}}^{x_{q}} \frac{d}{d x} \phi(x) \frac{1}{\varepsilon(x)} \frac{d}{d x} \psi(x) d x \\
V^{q}=-\int_{x_{q-1}}^{x_{q}} \phi(x) \psi(x) d x \\
W^{q}=\int_{x_{q-1}}^{x_{q}} \phi(x) \frac{1}{\varepsilon(x)} \psi(x) d x
\end{gathered}
$$

\section{i.2 Algebraic eigensystem}

In finite elements methods, the algebraic eigen-system from which eigenvalues and eigenvectors are sought is obtained by assembling element specific matrices. The present subsection is devoted to the building of such matrices. In element $\Omega^{j}, H_{y}^{j}$ and $E_{x}^{j}$ are represented as

$$
\begin{aligned}
& H_{y}^{j}(x)=\sum_{n=1}^{N^{j}+1} H_{y, n}^{j} B_{n}(u) \\
& E_{x}^{j}(x)=\sum_{n=1}^{N^{j}} E_{x, n}^{j} B_{n}(u)
\end{aligned}
$$

Generally, spectral finite elements are based on a set of Lagrange polynomials utilizing a specific nodal distribution on the interval $[-1,1]$ [7]. The originality of our approach lies in the chosen set of basis functions $B_{m}(u)$, which are build from Legendre polynomials in the following way:

$$
B_{m}(u)= \begin{cases}0.5\left(L_{0}-L_{1}\right), & m=1 \\ 0.5\left(L_{0}+L_{1}\right), & m=2 \\ L_{m}(u)-L_{m-2}(u), & m>=2\end{cases}
$$

where $L_{m}$ is the Legendre polynomial of degree $m$. The reduced variable

$$
u=\frac{2}{\Delta_{l}}\left(x-\Delta_{l}\right), \quad \Delta_{l}=x_{l}-x_{l+1}
$$

results from the mapping of the element $\Omega^{j}$ to the unit interval $[-1,1]$ where the Legendre polynomials are defined. The above basis sketched in Fig 2 was already used in [10] . It allows a straightforward assembly of elementary matrices in view of the building of the global matrix. Please, note that $H_{y}$ expansion contains one more basis function than that of $E_{x}$. This comes from the need to enforce the continuity of $H_{y}$ at the border of elements during the assembly process. Let us denote by $\mathbf{P}^{\mathbf{q}}$ the transformation matrix that gives the new defined basis in terms of the Legendre polynomials. In order to make the further assembly process more readable,
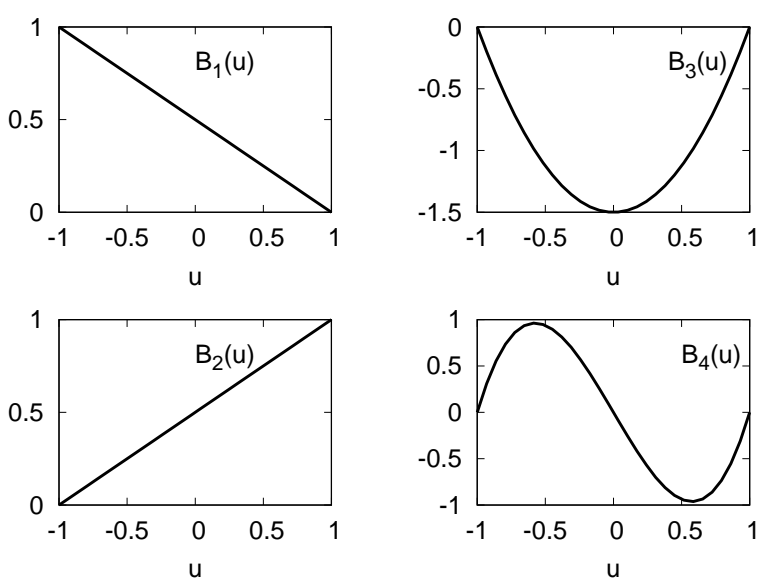

Figure 2: Illustration of the first four modified Legendre polynomials

we swap the second and the last element in the set of $B_{m}$. For a basis with $N^{q}+1$ elements, $\mathbf{P}^{q}$ is a square matrix of size $N^{q}+1$ which writes:

$$
\mathbf{P}^{\mathbf{q}}=\left[\begin{array}{cccccc}
.5 & -1 & 0 & \cdots & 0 & .5 \\
-.5 & 0 & -1 & \vdots & 0 & .5 \\
0 & 1 & 0 & \ddots & 0 & 0 \\
0 & 0 & 1 & \ddots & -1 & 0 \\
0 & 0 & 0 & \ddots & 0 & 0 \\
0 & 0 & 0 & 0 & 1 & 0
\end{array}\right]
$$

Now, we have to choose the testing functions $\phi_{m}(x)$. A common choice is to take also the $B_{m}(u)$. Thus, we are now able to express the elements of matrices $\mathbf{U}^{q}, \mathbf{V}^{q}$ and $\mathbf{W}^{q}$ :

$$
\begin{aligned}
U_{m n}^{q} & =\frac{2}{\Delta_{j}} \int_{-1}^{1} \frac{d B_{m}}{d u} \frac{1}{\varepsilon(u)} \frac{d B_{n}}{d u} d u \\
V_{m n}^{q} & =\frac{\Delta_{j}}{2} \int_{-1}^{1} B_{m} B_{n} d u \\
W_{m n}^{q} & =\frac{\Delta_{j}}{2} \int_{-1}^{1} B_{m} \frac{1}{\varepsilon(u)} B_{n} d u
\end{aligned}
$$

The last task is to compute the above elements. We restrict our analysis to the case where $\varepsilon(u)$ is constant and we denote by $\varepsilon^{j}$ the value of $\varepsilon(u)$ in element $\Omega^{j}$. Recall that the Legendre polynomials are orthogonal and that

$$
\int_{-1}^{1} L_{m} L_{n} d u=\frac{2}{2 m+1} \delta_{m n}
$$

with $\delta_{m n}$ the Kronecker symbol. Let us denote by $\mathbf{D}^{q}$ the diagonal matrix whose elements are the $2 /(2 m+1), m=$ $0, \cdots Q$. The calculation of elementary matrices $\mathbf{V}^{q}$ and $\mathbf{W}^{q}$ 
is straightforward and gives.

$$
\mathbf{V}^{q}=\frac{\Delta_{q}}{2}\left(\mathbf{P}^{q}\right)^{T} \mathbf{D}^{q} \mathbf{P}^{q}, \quad \mathbf{W}^{q}=\frac{1}{\varepsilon^{q}} \mathbf{V}^{q}
$$

where the letter $T$ designates the transposition.

The calculation of $\mathbf{U}^{q}$ is slightly more complicated. It makes use of the matrix Dr formed by the expansion coefficients of the derivative of the Legendre polynomials onto the Legendre polynomials themselves, that is:

$$
\frac{d L_{m}}{d u}=\sum_{n=0}^{N} D_{r m n} L_{n}(u)
$$

The derivation of matrix Dr is carried over to appendix A. Let us introduce the matrix $\dot{\mathbf{P}}^{q}$ corresponding to the expansion of the derivative of the $B_{m}(u)$ onto the Legendre polynomials. We have:

$$
\dot{\mathbf{P}}^{q}=\operatorname{Dr}^{q} \mathbf{P}^{q}
$$

The above described matrices allow the calculation of elementary matrix $\mathbf{U}^{q}$ in the form:

$$
\mathbf{U}^{q}=\frac{\Delta_{q}}{\varepsilon^{q}}\left(\dot{\mathbf{P}}^{q}\right)^{T} \mathbf{D}^{q} \dot{\mathbf{P}}^{q}
$$

\section{i.3 Assembly}

The next step in the finite element procedure is the assembly of elemental matrices in order to satisfy (9) with the matrices given by (19) and (22). Let us assume that the computational domain is divided into $Q$ elements with nodes labeled from 0 to $Q$ giving rise to elemental matrices with dimension $N^{q}+1$, $q=1,2, \cdots Q$. For periodic structures, the dimension of the global matrix is $N=\sum_{q=1}^{Q} N^{q}$. Let us consider a node $q$, $q=1, \cdots, Q-1$ which is shared by elements $\Omega^{q}$ and $\Omega^{q+1}$. The value of $H_{y}$ at node $q$ is simultaneously the $\left(N^{q}+1\right)$ th expansion coefficients in element $\Omega^{q}$ and the first one in element $\Omega^{q+1}$. We go from local numbering $p$ in the $P$ th element $\Omega^{p}$ to global numbering $n$ in the whole computational domain by:

$$
n=p+\sum_{q=1}^{Q}\left(N^{q}+1\right)-(Q-1)
$$

Hence, in the global matrix, the $N^{p}+N^{p+1}$ non-null elements on $n$th line corresponding to node $p$ writes:

$$
\begin{aligned}
X_{N^{p}+1,1}^{p}, X_{N^{p}+1,2}^{p} & \cdots, \\
& \\
& \left.X_{N^{p}+1, N^{p}+1}^{p}+X_{11}^{p}\right), \\
& \cdots, X_{1,2}^{p+1}, X_{1,3}^{p+1}, X_{1, N^{p+1}+1}^{p+1}
\end{aligned}
$$

where the $\mathbf{X}^{q}, q \in(p, p+1)$ refers to any of elemental matrices $\mathbf{U}^{q}, \mathbf{V}^{q}$ or $\mathbf{W}^{q}$.

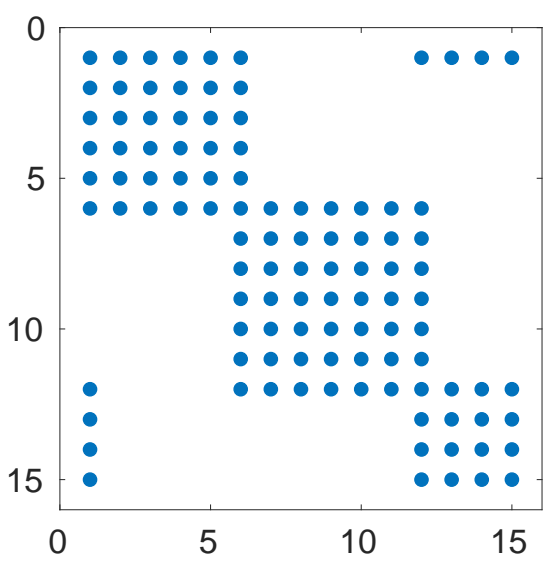

Figure 3: Example of assembly process of three elementary matrices for the solution of eigen-vector equation 6 with pseudo-periodic boundary conditions. The cardinality of elements is 6,7 and 5 respectively.

\section{i.4 Bloch periodic boundary conditions}

For the grating under consideration, the Bloch periodic boundary conditions stipulate that $H_{y}$ satisfies

$$
H_{y}(x+d)=\tau H_{y}(x), \quad \tau=\exp \left(-i \alpha_{0} d\right)
$$

The above particular boundary condition can be easily enforced. Indeed, it is enough to replace in element $\Omega^{Q}$ the last expansion basis function $B_{N Q+1}^{Q}$ by $\tau B_{1}^{1}$. Here, the exponents 1 and $Q$ refer to the element to which the basis function belongs. The set of test functions has to be modified accordingly: the test function $B_{N Q_{+1}}^{Q}$ is replaced by $\tau^{*} B_{1}^{1}$ where * designates the complex conjugation. One can see that $I_{N}$ and $I_{0}$ in (9) are equal and thus disappear in the summation. Moreover, only the last line and the last column of $\mathbf{X}^{Q}$ are modified. Let us denote by $\mathbf{F}$ the global matrix built from the matrices $\mathbf{X}^{q}$. Enforcing the Bloch periodic boundary conditions consists in doing the three following operations

$$
\begin{aligned}
& F_{11}=X_{11}^{1}+X_{N^{Q}+1 N^{Q}+1}^{Q} \\
& \left(F_{1 N-N}, F_{1, N-N} Q_{+1}, \cdots, F_{1, N}\right)= \\
& \tau^{*}\left(X_{N^{Q} 1_{1}}^{Q}, X_{N^{Q} 2_{2}}^{Q}, \cdots, X_{N N_{N}}^{Q}\right) \\
& \left(F_{N-N Q}{ }_{1}, F_{N-N Q+11}, \cdots, F_{N 1}\right)= \\
& \tau\left(X_{1 N^{Q}}^{Q}, X_{2 N Q^{Q}}^{Q}, \cdots, X_{N Q N Q}^{Q}\right)
\end{aligned}
$$

Fig 3 illustrates the filling of the global matrices $\mathbf{U}, \mathbf{V}$ and $\mathbf{W}$ for three elements and $N^{q}=5,6,4$ respectively. Finally, we have transformed (2) into a generalized algebraic eigenvector equation;

$$
(\mathbf{U}+\mathbf{V}) \mathbf{H}_{\mathbf{y}}=\gamma^{2} \mathbf{W} \mathbf{H}_{\mathbf{y}}
$$




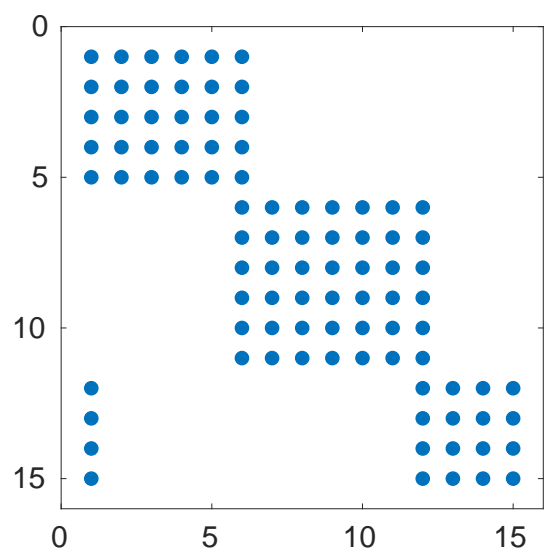

Figure 4: Example of assembly process of three elementary matrices for the derivationn of the $E_{x}$ electric field component given by Eq32 $H_{y}$ has 6,7 and 5 spectral coefficient in elements $\Omega^{1}, \Omega^{2}$ and $\Omega^{3}$, respectively whereas $E_{x}$ has one less.

\section{ii. $E_{x}$ Component}

In a simple spectral problem, we would not necessarily compute the $E_{x}$ component. In the present problem, we have to determine the constant coefficients $A_{l n}^{ \pm}$, which requires to enforce Dirichlet boundary conditions for $H_{y}$ and $E_{x}$ at $z=0$ and $z=h$. Let us consider an eigen vector in element $\Omega^{q}$. For the sake of conciseness, in what follows, we do not make any reference neither to the region nor to the number of the eigenvector. From (1b) and (13) we have:

$$
\sum_{n=1}^{N^{q}} E_{x n}^{q} B_{n}(u)=\frac{\gamma}{\omega \varepsilon^{q}} \sum_{n=1}^{N^{q}+1} H_{y n}^{q} B_{n}(u)
$$

In a second step, (28) is projected onto the set of basis function $B_{m}, m=1, \cdots, N^{q}$ which gives:

$$
\begin{gathered}
\sum_{n=1}^{N^{q}} E_{x n}^{q} \int_{-1}^{1} B_{m}(u) B_{n}(u) d u= \\
\frac{\gamma}{\omega \varepsilon^{q}} \sum_{n=0}^{N^{q}} H_{y n}^{j} \int_{-1}^{1} B_{m}(u) B_{n}(u) d u
\end{gathered}
$$

Let us introduce the matrix $\tilde{\mathbf{P}} q$ obtained by removing the $N^{q}$ th column from $\tilde{\mathbf{P}}^{q}$. Hence $\tilde{\mathbf{P}}^{q}$ has $N^{q}+1$ rows and $N^{q}$ columns. Similarly let us define matrix $\tilde{\mathbf{D}}$ by removing the $\left(N^{q}+1\right)$ th column and the $\left(N^{q}+1\right)$ th line from matrix $\tilde{\mathbf{D}}$. In matrix form, (29) writes:

$$
\mathbf{E}_{\mathbf{x}}{ }^{q}=\mathbf{Z}^{q} \mathbf{H}_{\mathbf{y}}{ }^{q}
$$

with

$$
\mathbf{E}_{\mathbf{x}}^{q}=\left[E_{x 1}^{N^{q}}, E_{x 2}^{N^{q}}, \cdots, E_{x N^{q}}^{N^{q}}\right]^{T}
$$

$$
\mathbf{Z}^{q}=\frac{1}{\omega \varepsilon^{q}}\left(\left(\tilde{\mathbf{P}}^{q}\right)^{T} \tilde{\mathbf{D}}^{q} \tilde{\mathbf{P}}^{q}\right)^{-1}\left(\left(\tilde{\mathbf{P}}^{q}\right)^{T} \mathbf{D}^{q} \mathbf{D}^{q}\right)
$$

Matrix $\mathbf{Z}^{q}$ has $N^{q}$ rows and $N^{q}+1$ columns. Let us denote by $\mathbf{Z}$ the global matrix that results from the assembly of elemental matrices $\mathbf{Z}^{q}$. It takes into account the continuity of $H_{y}$ at the borders of elements in the following way: the $n$th column in matrix $\mathbf{Z}$ corresponding to the connection between two consecutive elements $\Omega^{p}$ and $\Omega^{p+1}$ is the concatenation of the last column of $\Omega^{p}$ and the first column of $\Omega^{p+1}$. Lastly, the quasi-periodicity of $H_{y}$ amounts to put the $N^{Q}$ entries of the last column of matrix $\mathbf{Z}^{\mathrm{Q}}$ multiplied by the quasi periodic coefficient $\tau$ at the end of the first column of matrix $\mathbf{Z}$. This process is illustarted in Fig 4

\section{iii. Special case of homogeneous media}

As discussed in [11], we use computed eigenvectors in every region, which allows matching the different fields at the boundary of the grating without additional projection. In homogeneous media 1 and 3, exact eigen-solutions are the wellknown Rayleigh expansions. In those media, the number of basis functions should be large enough so that any computed real eigenvalues, when they exist, can be associated to a diffracted order. In that case, provided that the eigenvalue is not degenerate, up to a multiplicative constant coefficient, the associated eigenvector tends to the vector formed by the expansion coefficient of the corresponding plane wave. Indeed, in a similar way to what was done in [11], we replace the computed eigenvectors associated to real eigenvalues, and only these ones, by their Rayleigh expansion counterpart. In other words, for any $\alpha_{q}=\alpha_{0}+q 2 \pi / d$ such that $-1 \leq \alpha_{q} \leq 1$, we expand $\exp \left(-i \alpha_{q} x\right)$ onto $B_{m}$ basis. Since the $P_{m}$ are themselves linear combination of Legendre polynomials, we first expand the exponential functions onto the latter:

$$
\exp \left(-i \alpha_{q} x\right)=\sum_{m=1}^{N^{p}+1} \tilde{\psi}_{m q}^{p} L_{m}(u(x)) \quad x_{p-1} \leq x \leq x_{p}
$$

Using the following relation

$$
\int_{-1}^{1} L_{n}(x) \exp (i \alpha x) d x=i^{n} \sqrt{\frac{2 \pi}{\alpha}} J_{n+1 / 2}(\alpha)
$$

and the orthogonality of Legendre polynomials we may derive:

$$
\tilde{\psi}_{m q}^{p}=(-i)^{m} \frac{2 m+1}{2} \sqrt{\frac{2 \pi}{\zeta_{q}}} J_{m+1 / 2}\left(\zeta_{q}^{p}\right) \exp \left(-i \zeta_{q}^{p}\right)
$$

with

$$
\zeta_{q}^{p}=\frac{\alpha_{q} \Delta_{p}}{2}
$$


Table 1: Parameters of investigated dielectric metallic gratings

\begin{tabular}{ccc}
\hline Parameter & Grating 1 & Grating 2 \\
\hline$\theta$ & 0 & 35 \\
$\lambda$ & .55 & .45 \\
$c$ & $.3 \times .25$ & .5 \\
$d$ & .25 & .2 \\
$h$ & .2 & .521 \\
$n_{3}$ & 1.5 & 1.5 \\
$n_{21}$ & 1 & 1 \\
$n_{22}$ & $3.18-4.41 j$ & $-\sqrt{25} j$ \\
\hline
\end{tabular}

The $\psi_{m q}^{p}$ such that:

$$
\exp \left(-i \alpha_{q} x\right)=\sum_{m=1}^{N^{p}+1} \tilde{\psi}_{m q}^{p} B_{m}(u(x)) \quad x_{p-1} \leq x \leq x_{p}
$$

are linked to the $\tilde{\psi}_{m q}^{p}$ by:

$$
\psi_{q}^{p}=\left(\mathbf{P}^{p}\right)^{-1} \tilde{\psi}_{q}^{p} \quad \psi_{q}^{p}=\left[\begin{array}{c}
\psi_{1 q}^{p} \\
\vdots \\
\psi_{N^{q}}^{p}
\end{array}\right] \quad \tilde{\psi}_{q}^{p}=\left[\begin{array}{c}
\tilde{\psi}_{1 q}^{p} \\
\vdots \\
\tilde{\psi}_{N^{q}}^{p}
\end{array}\right]
$$

\section{Results}

In this section, we validate our code by comparing our results with already published data. The parameters of the considered gratings are collected in table 1 In the past those cases were investigated because convergence problems or instabilities occurred when the Fourier Modal Method was used to analyse them. Let us first consider Grating 1 which was studied in [12], [13] and [14]. We have first to verify that our method converges to the same value as others methods. For that purpose we have plotted computed efficiencies as a function of the inverse of the truncation number which is defined as the dimension of the matrix from which eigenvalues are sought. Fig 5 illustrate the convergence of the zeroth transmitted order computed with the SEM and with the Fourier Matching Pseudo Spectral Modal Method (PSMM) [16], [11]. We found that both converged to $T_{0}=.698305$ which becomes the assumed "exact" value. The same figure also shows the error in the computation of $T_{0}$ defined as:

$$
E r r=\log \left(\left|T_{0}-T_{\text {Dexact }}\right|\right)
$$

We made the same kind of simulations for a lossless metallic grating for which numerical data was available [14], [15]. By running our codes, including Fourier Modal Method with Adaptive Spatial Resolution, (ASR-FMM), [17], with huge truncation orders, we found that the "exact" zeroth reflected

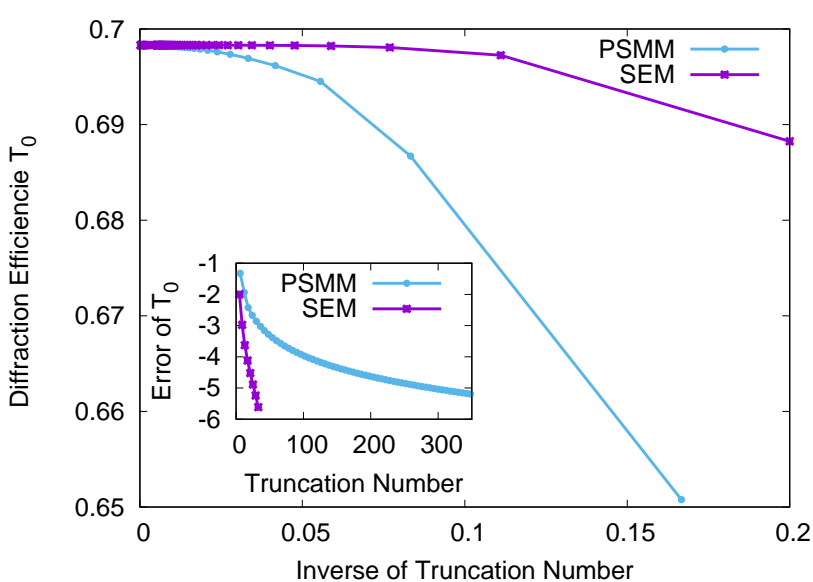

Figure 5: Zeroth transmitted order of grating 1 against the inverse of the truncation order computed with SEM and with PSFM. It is observed that both methods converge to the same value. The inset gives the error of $T_{0}$.

order was $R_{0}=.127698$. The computed values are summarized in table 2 and table 3

Table 2: Specular Reflected Efficiency for the lossless grating 2 computed with different modal methods.TN is the dimension of the matrix from which eigenvalues are sought.

\begin{tabular}{llll}
\hline TN & SEM & PSMM[14] & AMM[15] \\
\hline 33 & $1.2955593 \mathrm{e}-01$ & $1.3059319(-1)$ & $1.2544877(-1)$ \\
65 & $1.2812373 \mathrm{e}-01$ & $1.2835611(-1)$ & $1.2694599(-1)$ \\
129 & $1.2779410 \mathrm{e}-01$ & $1.2787523(-1)$ & $1.2745205(-1)$ \\
257 & $1.2771968 \mathrm{e}-01$ & $1.2771968(-1)$ & $1.2761832(-1)$ \\
513 & $1.2770294 \mathrm{e}-01$ & $1.2769345(-1)$ & $1.2767238(-1)$ \\
1025 & $1.2769926 \mathrm{e}-01$ & $1.2768770(-1)$ & $1.2768988(-1)$ \\
\hline
\end{tabular}

From a practical view point, it is of course unnecessary to use such large truncation numbers. On the one hand, our aim is to verify the stability and the robustness of the method and on the other hand to determine a numerical "exact" value that allows to calculate an error.

The convergence of any modal methods applied to gratings depends on the accuracy with which eigenvalues and eigen modes are computed on the one hand and on the matching of plane waves at the border of the grating region on the other hand. Or, for lamellar grating, the junction between the grating region itself and the homogeneous regions in which it is embedded creates a wedge with three different materials and a right angle. It is well-known that in Tm polarization the transverse components of the electric field and magnetic field may be singular at the sharp edge of the wedge. When the singularity is not incorporated into the algorithms as is the case in this paper, the accuracy of the 
Table 3: Specular Reflected Efficiency for the lossless grating 2. Benchmark between three in-house codes with huge truncation order. $T N$ is the dimemsion of the matrix from which eigenvalues are sought.

\begin{tabular}{llll}
\hline TN & SEM & PSMM & ASR-FMM \\
\hline 33 & $1.2955593(-1)$ & $1.30801852(-1)$ & $1.2357814(-1)$ \\
65 & $1.2812373(-1)$ & $1.2844758(-1)$ & $1.2776491(-1)$ \\
129 & $1.2779410(-1)$ & $1.2787316(-1)$ & $1.2772640(-1)$ \\
257 & $1.2771968(-1)$ & $1.2773789(-1)$ & $1.2769891(-1)$ \\
513 & $1.277029(-1)$ & $1.2770631(-1)$ & $1.2769842(-1)$ \\
1025 & $1.2769926(-1)$ & $1.2770043(-1)$ & $1.2769835(-1)$ \\
1200 & $1.27698951(-1)$ & $1.2769961(-1)$ & $1.2769824(-1)$ \\
1500 & $1.2769867(-1)$ & $1.2769905(-1)$ & $1.2769812(-1)$ \\
1800 & $1.2769851(-1)$ & $1.2769879(-1)$ & $1.2769809(-1)$ \\
2049 & $1.2769844(-1)$ & $1.2769864(-1)$ & $1.2769814(-1)$ \\
2401 & $1.2769838(-1)$ & $1.2769854(-1)$ & $1.2769821(-1)$ \\
3001 & $1.2769831(-1)$ & $1.2769846(-1)$ & $1.2769821(-1)$ \\
4097 & $1.2769826(-1)$ & $1.2769839(-1)$ & $1.2769817(-1)$ \\
\hline
\end{tabular}

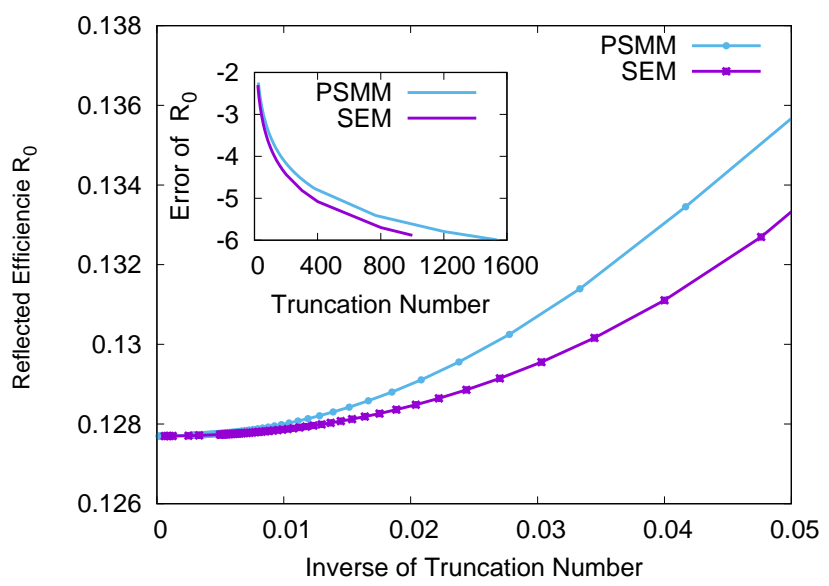

Figure 6: Specular reflected order of grating 2 against the inverse of the truncation order computed with SEM and with PSFM. It is observed that both methods converge to the same value. The inset gives the error of $R_{0}$.

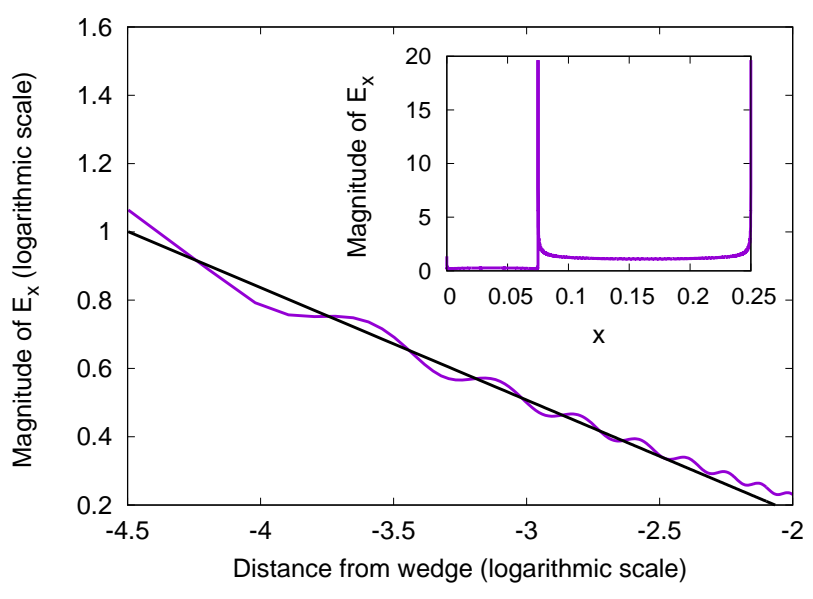

Figure 7: Magnitude of $E_{x}$ at the upper surface of grating 1. The straight line fits the data between -4.5 and -3 , that is close to the wedge. Its equation is $y=(.3291 \times x-.48)$.

numerical method can be tested by verifying whether computed data behaves as predicted in the vicinity of singular points. With reference to Fig 1 it can be shown that at $z=h$, $x>c, E_{x}$ has the following asymptotic form:

$$
E_{x}=A \times(x-c)^{\tau-1}
$$

where $x-c$ is the distance from the sharp edge. The singularity exponent $\tau$ has the following expression in the special case where $n_{1}=n_{21}$ [18]:

$$
\tau=\frac{2}{\pi} \arcsin \left(1-\frac{1}{4} \frac{n_{22}^{2}-n_{1}^{2}}{n_{22}^{2}+n_{1}^{2}}\right)^{1 / 2}
$$

For grating 1, we have $\tau=.666+.0335 i$ and for grating 2 $\tau=.635$. The smaller the $\tau$ value, the larger the truncation number. Indeed the comparison of Fig 5 and Fig 6 shows that the numerical convergence of the efficiencies is faster for grating 1 than for grating 2 . However, the difference in the value of $\tau$ is probably not the main reason that explains the speed of convergence between the two gratings configurations. In Fig, 7 the magnitude of $E_{x}$ is plotted against the distance from the wedge in logarithmic scale, for a truncation order of 300. The distance is expressed in multiple of the wavelength. Fitting the plot with a straight line of the form $y=a \times x+b$ between -4.5 and -3 gives $a=.33$ in very good accordance with the theoretical value .3340 . Other simulations, not shown here, with lower truncation orders give the same behavior but the parameter $a$ is smaller and varies around 0.29 . One can conclude that the method reproduces with a satisfactory accuracy the near field. 


\section{Conclusion}

We have developed a code based on the spectral element method in which hierarchical basis functions derived from Legendre polynomials were introduced. The code has been validated by comparison with other well established methods on cases for which obtaining numerical convergence was considered challenging. On these examples, the SEM demonstrated accuracy and stability. The formulation which we have just presented enriches the broad range of the numerical modal methods. However compared to the pioneering work of Morf [2], who was the first to use Legendre polynomials expansions in numerical modal methods, it is our opinion that the SEM is simpler to implement. In the future, it would be interesting to benchmark honestly the many numerical methods with appropriate metrics in order to better understand their optimal field of use.

\section{APPENDIX A: DERIVATION OF THE DERIVATIVE OPERATOR ASSOCIATED WITH LEGENDRE POLYNOMIAL EXPANSIONS}

Consider the expansion of functions $F(u)$ and its derivative $G(u)$ onto the set of $N+1$ Legendre polynomials :

$$
\begin{aligned}
& F(u)=\sum_{n=0}^{N} F_{n} L_{n}(u) \\
& \frac{d}{d u} F(u)=G(u)=\sum_{m=0}^{N} G_{m} L_{m}(u)
\end{aligned}
$$

We seek the elements of the $\mathbf{D}$ matrix which relate the expansion coefficients of the derivative of the $F(u)$ function to the coefficients of the function itself that is:

$$
G_{m}=\sum_{n=0}^{N} D_{r m n} F_{n}
$$

by deriving $F(u)$, we are led to:

$$
\sum_{n=0}^{N} F_{n} \frac{d}{d u} L_{n}(u)=\sum_{n=0}^{N} G_{n} L_{n}(u)
$$

The projection of the above equation onto Legendre polynomial $L_{m}(u)$ gives:

$$
\begin{gathered}
\sum_{n=0}^{N} F_{n} \int_{-1}^{1} L_{m}(u) \frac{d}{d u} L_{n}(u) d u=\sum_{\substack{n=0 \\
m, n}}^{N} G_{n} \int_{-1}^{1} L_{m}(u) L_{n}(u) d u \\
\end{gathered}
$$

Taking into account the two following properties of Legendre polynomials:

$$
\int_{-1}^{1} L_{m}(u) \frac{d}{d u} L_{n}(u) d u=\left\{\begin{array}{l}
2 \text { if } n-m>0 \text { and } m-n \text { odd } \\
0 \text { otherwise }
\end{array}\right.
$$

and

$$
\int_{-1}^{1} L_{m}(u) L_{n}(u) d u=\frac{2}{2 m+1} \delta_{m n}
$$

where $\delta_{m n}$ is the Kronecker symbol we get:

$$
D_{r m n}=\frac{2 m+1}{2} d_{m n}
$$

with

$$
d_{m n}= \begin{cases}\left(1-(-1)^{n-m}\right), & m, n \in\{0,1, \cdots N\}, \\ 0 \text { otherwise } & n-m \text { odd }, n>m\end{cases}
$$

\section{AcKNOWLedgments}

This work was sponsored by a public grant overseen by the French National Agency as part of the "Investissements d'Avenir" through the IDEX-ISITE initiative CAP 2025 (ANR-16-IDEX-0001).

\section{Disclosures}

The authors declare no conflicts of interest.

\section{REFERENCES}

[1] M. G. Moharam and T. K. Gaylord, "Diffraction analysis of dielectric surface-relief gratings," J. Opt. Soc. Am A,vol.72, p. 1385-1392 (1982).

[2] R. H. Morf, "Exponentially convergent and numerically efficient solution of Maxwell's equations for lamellar gratings," J. Opt. Am. A, vol. 12, p. 1043-1056 (1995).

[3] K. Edee, "Modal method based on sub-sectional Gegenbauer polynomial expansion for lamellar grating," J. Opt. Soc. Am A, vol.28, p. 2006-2013 (2011).

[4] K.Edee,I.Fenniche, G.Granet, and B.Guizal, "Modal method based on subsectional Gegenbaueur polynomial expansion for lamellar gratings: weighting function, convegence and stability", PIER, vol.133, p.1735 (2012)

[5] M. H. Randriamihaja, G. Granet, K. Edee,and K. Raniriharinosy, "Polynomial modal analysis of lamellar diffraction gratings in conical mounting," J. Opt. Soc. Am. A, vol. 33, p. 1679-1686 (2016).

[6] E. Popov, (Ed.), "Gratings:Theory and Numeric Applications,"Institut Fresnel,CNRS,AMU, (2012). 
[7] C. Pozirikidis, 2014, "Introduction to Finite and Spectral Element Methods Using Matlab,"CRC Press, (2014).

[8] A. C. Polycarpou, "Introduction to the Finite Element Method in Electromagnetics," Morgan and Claypool Publisers'series Synthesis lectures on computational electromagnetics

[9] J. L. Volakis, A. Chatterje, and L. C. Kempel, "Finite Element Method for Electromagnetics,Antennas, Microwave circuits, and scattering applications," IEEE press.

[10] E. Jorgensen, J. L. Volakis, P. Meincke, and O. Breinbjerg, "Higher Order Hierarchical Legendre Basis Functions for Electromagnetic Modeling," IEEE Trans. Antennas Propagat., vol.52 p. 2985-2994 (2004).

[11] G. Granet, "Fourier-matching pseudospectral modal method for diffraction gratings:comment," J. Opt. Soc. Am. A,vol.29, p. 1843-1845 (2012).

[12] S. Peng and G. M. Morris, 1995, "Efficient implementation of rigorous coupled-wave analysis for surface relief gratings," J. Opt. Soc. Am. A, vol.12, p. 1087-1096.

[13] P. Lalanne and G. M. Morris, "Highly improved convergence of the coupled-wave method for TM polarization," J. Opt. Soc. Am. A, vol.13, p. 779-784 (1996).

[14] Y.-P. Chiou, W.-E. Yeh,and N.-Y. Shih, 2009, "Analysis of Hightly Conducting Lamellar Gratings With Multidomain Pseudospectral Method," J. Lightwave Technol., vol.27, p. 5151-5159 (2009).

[15] M. Foresti, L. Menez, and A. V. Tishchenko, "Modal method in deep metal-dielectric gratings:the decisive role of hidden modes," J. Opt. Soc. Am. A, vol.23, p. 2501-2509 (2006).

[16] D. Song,L. Yuan, and Y. YLu, 2011, "Fourier-matching pseudospectral modal method for diffraction gratings," J. Opt. Soc. Am. A, vol.28, p. 613-620 (2011).

[17] G. Granet, 1999, "Reformulation of the lamellar grating problem through the concept of adaptive spatial resolution," J. Opt. Soc. Am. A, vol.16, p. 2510-2516 (1999).

[18] L. Li and G. Granet, "Field singularities at lossless metaldielectric right-angle edges and their ramifications to the numerical modeling of gratings," J. Opt. Soc. Am. A, vol.28, p. 738-746 (2011). 